\title{
Femtosecond quantum dynamics of excited-state evolution of halide perovskites: Quantum chaos of molecular cations
}

\section{Yeonghun Lee}

University of Texas at Dallas

Hyungjun Kim

Korea Advanced Institute of Science and Technology

Ki-Ha Hong

Hanbat National University https://orcid.org/0000-0001-7457-8196

Kyeongjae Cho ( $\square$ kjcho@utdallas.edu )

The University of Texas at Dallas https://orcid.org/0000-0003-2698-7774

\section{Article}

Keywords: excited-state quantum dynamics, organic cation, hybrid perovskites, TDDFT

Posted Date: September 21st, 2020

DOI: https://doi.org/10.21203/rs.3.rs-78504/v1

License: (c) (i) This work is licensed under a Creative Commons Attribution 4.0 International License. Read Full License

Version of Record: A version of this preprint was published at The Journal of Physical Chemistry C on May 7th, 2021. See the published version at https://doi.org/10.1021/acs.jpcc.1c01439. 


\title{
Femtosecond Quantum Dynamics of Excited-State
}

\section{Evolution of Halide Perovskites: Quantum Chaos of}

\section{Molecular Cations}

\author{
Yeonghun Lee ${ }^{1}$, Hyungjun $\mathrm{Kim}^{2}$, Ki-Ha Hong ${ }^{3, *}$, and Kyeongjae Cho ${ }^{1, *}$ \\ ${ }^{1}$ Department of Materials Science and Engineering, University of Texas at Dallas, 800 West \\ Campbell Road, Richardson, TX 75080, USA \\ ${ }^{2}$ Department of Chemistry, Korea Advanced Institute of Science and Technology, 291 Daehak- \\ ro, Yuseong-gu, Daejeon 34141, Republic of Korea \\ ${ }^{3}$ Department of Materials Science and Engineering, Hanbat National University, 125 Dongseo- \\ daero, Yuseong-Gu, Daejeon, 34158, Republic of Korea
}

\begin{abstract}
AUTHOR INFORMATION
*Correspondence and requests for materials should be addressed to K.-H.H. (email: kiha.hong@ hanbat.ac.kr) or to K.C. (email: kjcho@utdallas.edu).
\end{abstract}




\begin{abstract}
The excited-state quantum dynamics of the organic cation in hybrid perovskites are investigated using the time-dependent density functional theory (TDDFT). The time-dependent non-adiabatic bond fluctuation behaviors reveal that the energy relaxation follows different pathways depending on the chemical bonding characteristics and energy transfer modes within the cation molecule, which can fundamentally affect its photostability. For the ammonium-group-containing cations, such as methylammonium (MA) or ethylammonium (EA), local vibrational modes survive for a long time. However, as their lowest unoccupied molecular orbital (LUMO) having $\pi^{*}$ characters, the amidinium-group-containing cations, such as formamidinium (FA) or guanidinium (GA), efficiently dissipate deposited energy via chaotic intramolecular vibrational energy redistribution (IVR). The distinct dynamic behaviors of A-site molecular cations are closely related to the quantum ergodicity, which can bring enhanced photochemical stability of FA and GA compared to MA and EA. Our theoretical investigation reveals the quantum chaos origin of better light stability of FA-based perovskites and serves the future research direction of the A-site engineering for better solar cells and light-emitting devices.
\end{abstract}


There has been tremendous progress in the various perovskite-based applications during the last ten years, such as solar cells (PeSCs) ${ }^{1-5}$, light-emitting diodes $(\mathrm{PeLEDs})^{6-8}$, resistive memories ${ }^{9,10}$, and photodetectors ${ }^{11,12}$. The certified power conversion efficiency (PCE) of PeSCs is already over $25 \%$ and the external quantum efficiency of PeLEDs exceeds $20 \%^{6,13}$. An organic cation is the unique feature of organic-inorganic hybrid perovskites enabling unusual materials properties. Methylammonium (MA) cation was the first adopted cation molecule to make solar cells by the Miyasaka group in $2009^{14}$. From the initial development stage of perovskite solar cells, various cation molecules are tested to develop more efficient and robust perovskite devices. Formamidinium (FA) cation has shown the most outstanding achievements among diverse A-site molecule candidates, which was first implemented in the solar cells by Snaith's group in $2014^{15}$. Ethylammonium (EA) and guanidinium (GA) cations have been tried even in early stage ${ }^{16,17}$, and other bigger cation molecules have also shown promising behaviors for the development of lowdimensional perovskites and 2D-3D hybrid perovskite structures ${ }^{18,19}$.

Organic cations are located at the $\mathrm{A}$-site of $\mathrm{ABX}_{3}$ type perovskite crystal structures. A-site cations play crucial roles in deciding the stabilities and carrier transport characteristics. FA has been considered a more robust molecular cation because empirically $\mathrm{FAPbI}_{3}$ has shown better thermal and photostability than $\mathrm{MA}^{20-22}$. FA exhibits a smaller dipole moment which can improve the stability against moisture ${ }^{23}$. Recently, there have been experimental reports presenting the enhanced stability by incorporating GA or EA cations as additives or dopants ${ }^{24,25}$.

Photostability can be the fundamental bottleneck for the commercialization of hybrid perovskites. The stability under illumination needs further progress than that of dark conditions despite recent fast improvements in the long term stability of perovskite solar cells ${ }^{26}$. A-site cations have shown a close relationship with light-induced instability. Nickel et al. reported that MA 
cations can be dissociated into $\mathrm{CH}_{3} \mathrm{NH}_{2}$ and hydrogen by UV light irradiation ${ }^{27}$. These authors have proposed a scenario that the photodissociation of MA is induced by the electron excitation from the valence band to the antibonding orbitals of MA molecules based on their gas effusion experimental data ${ }^{27}$. Similar photodissociation studies have been reported for various experimental conditions $^{28,29}$, and even in the case of layered perovskites ${ }^{30}$.

It is also found that A-site molecular cations can affect the dynamics of excited carriers as well. Chen et al. recently reported that hot carrier (HC) cooling has cation-dependence, and the A-site FA shows the fastest $\mathrm{HC}$ cooling among FA, MA, and Cesium cations ${ }^{30}$. It was proposed that a slow charge-carrier cooling can give the chance to extract hot-carrier, enhancing photovoltaic efficiency ${ }^{31}$.

These findings show that excited-state dynamics of A-site cations have a close relationship with photostability and $\mathrm{HC}$ cooling. However, most theoretical studies on the dynamics of the excited carrier have focused on the interaction between the electron and inorganic-lattice phonon through time-domain density functional theory, where the ionic motion is limited in an equilibrium state ${ }^{32-}$ 34. The effects of carrier injection into A-site cation molecules will introduce transient electronion non-equilibrium dynamics over attosecond-femtosecond time scales, but has not been clearly examined although the direct evidence was proven by the Nickel et al.'s experiment ${ }^{27}$. Such an excited electron dynamics study is beyond the capacity of usual DFT simulations and has not been clearly examined yet to elucidate the underlying mechanisms of excited electron-ion dynamics of A-site molecules in the hybrid perovskites.

This study aims to investigate the impacts of excited-state evolution with attosecond resolution for various A-site molecules for the femtosecond ranges through the state-of-the-art time- 
dependent density functional theory (TDDFT) calculations ${ }^{35,36}$. Calculated dynamic behaviors are closely correlated with the photostability and hot carrier dynamics of halide perovskites. From the fundamental analysis of excited electron-ion quantum dynamics, quantum chaos ${ }^{37-39}$ and quantum ergodicity ${ }^{40}$ are found to play prominent roles in determining the photostability of hybrid perovskites with different A-site cations.

\section{Results}

Model description. Fig. 1a represents an ideal cubo-octahedral lattice structure of $\mathrm{ABX}_{3}$ type perovskites. A distinct feature of organic-inorganic hybrid perovskites is that A-site cation can be made of various molecules. It is well known that the valence band maximum (VBM) state and conduction band minimum (CBM) state mainly consist of I $5 p$ and $\mathrm{Pb} 6 p$ orbitals, respectively. Furthermore, the A-site cations have molecular orbitals within the valence and conduction bands, away from the band gap (i.e., LUMO state above the conduction band edge and HOMO state below the valence band edge). Under light illumination, valence band edge electrons are excited to conduction band states for photo-carrier generation, and also to A-site LUMO states with high energy photon absorption. Such photoexcitation of valence electrons into LUMO states of A-site molecules can be modeled with A (= MA, EA, FA, GA)-I molecules, as shown in the right panel of Fig. 1a. Hereafter, we refer to cations- $\mathrm{MA}^{+1}, \mathrm{EA}^{+1}, \mathrm{FA}^{+1}$, and $\mathrm{GA}^{+1}$-as MA, EA, FA, and GA for simplicity unless otherwise stated. Fig. 1b describes how various excitations induced by light absorption can come down to electron capture into the LUMO state of the cations: (1) an electron is directly excited from the VBM to the LUMO; (2) a higher energy orbital is occupied first by an excited electron, and subsequent electronic relaxation rapidly occurs similar to Kasha's 
rule $^{41}$; (3) the LUMO is occupied by an electron that travels in the CB of an inorganic cell after photoexcitation; (4) we cannot exclude the possibility of excitation from filled trap states within band gap such as $\mathrm{I}_{\mathrm{i}}, \mathrm{I}_{\mathrm{MA}}$, and $\mathrm{Pb}$ dimer induced by $\mathrm{V}_{\mathrm{I}}^{42,43}$. The excitation is accompanied by creating a highly delocalized hole in the valence band, which, however, hardly influences the Asite molecule electronic structure due to the delocalized wavefunctions in periodic solids. Therefore, the molecular systems with an additional electron- $\mathrm{MAI}^{-1}, \mathrm{EAI}^{-1}, \mathrm{FAI}^{-1}$, and $\mathrm{GAI}^{-1}$ are selected to model the light-induced degradation originating from the A-site cation instability (after a model validation by comparing with A-site molecules in crystalline structure as shown in Supplementary Notes 1). During electron-ion dynamics simulations, the position of an iodine atom keeps unchanged representing the rigidity of I ions in the lattice structure. The A-I molecule model is a reasonable model approximation in which atoms forming A-site molecules are relatively free to move within the framework of iodine atoms forming strong covalent bonds to maintain the perovskite structure.

Our non-adiabatic dynamics simulation begins with the electron capture into the LUMO state of A-site molecule because the LUMO-occupied state most likely governs the pertinent molecular dynamics. Even if we start with an electron in higher energy LUMO+ $\alpha$ state of A-site molecule, the electron rapidly comes down to the LUMO according to Kasha's rule, and the molecule readily reaches the local electronic ground state. Ionic energy deposited during this initial relaxation process will be redistributed in accordance with the LUMO-occupied electronic state. When subsequent ionic energy deposition can occur in any form, the stored energy or any temporary localized vibration will be redistributed following the LUMO-occupied electronic state as well. Accordingly, for an extensively long time, the LUMO character plays a prominent role in molecular dynamics. 
a

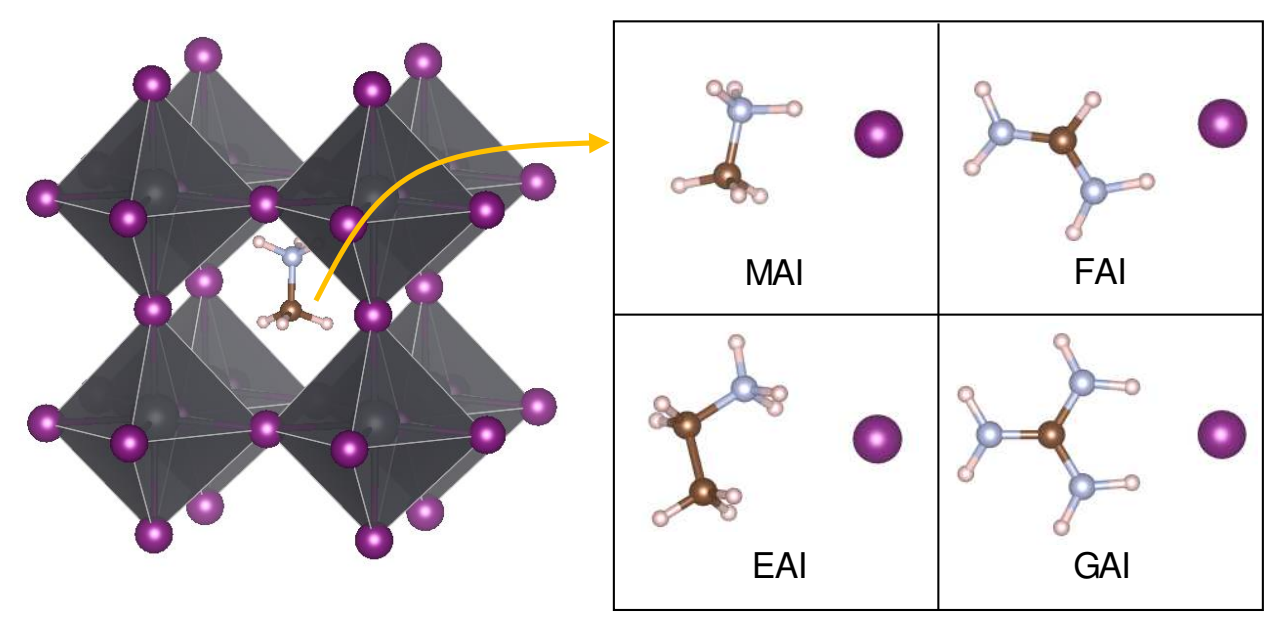

$\odot \mathrm{Pb} \odot \mathrm{I} \odot \mathrm{H} \quad \circ \mathrm{N} \odot \mathrm{C}$

b

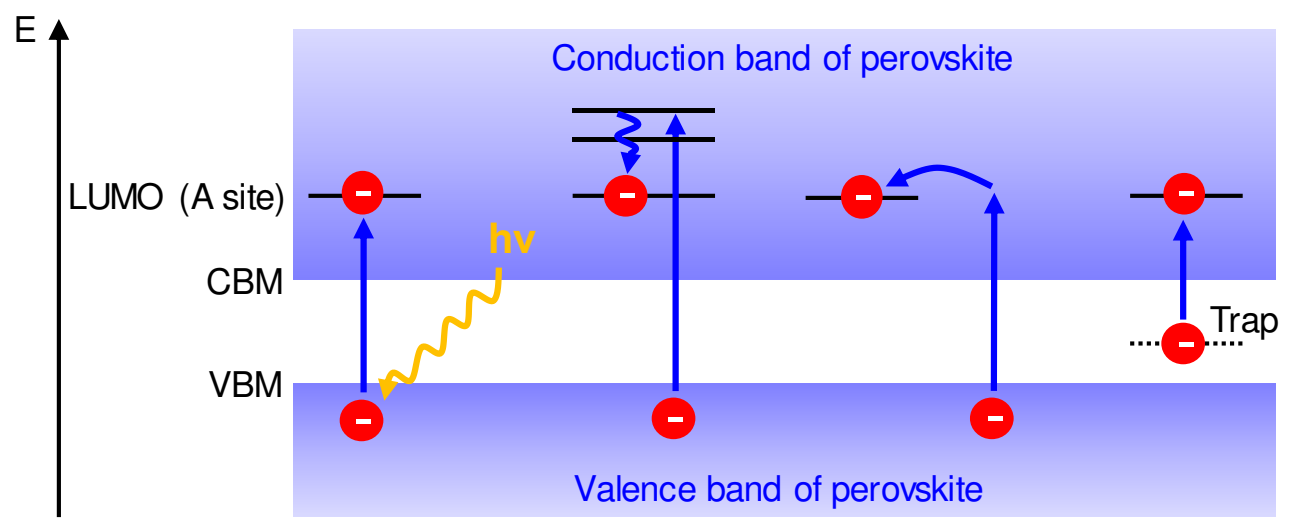

Fig. 1 a Atomistic structure of organic-inorganic hybrid perovskites (left). To study the lightinduced dynamics of A-site organic cations in the perovskites, we consider molecular systems of an organic cation attached to an iodine anion (right). b Schematic of electron capture processes into the LUMO of A-site cations. Energy levels of three-dimensional (3D) perovskites and molecules are represented by shaded regions and solid lines, respectively. A dotted line represents a trap state. 


\section{Dynamics simulation of ammonium group (AMG) and amidinium group (ADG). The atomic}

vibration induced by a carrier excited into the LUMO is calculated during $300 \mathrm{fs}$, and the time evolution of C-N and N-H bond lengths are represented in Fig. 2 (full details of dynamics are shown in Fig. S3). For the detailed analysis of electron-ion dynamics, we selected the most sensitive N-H bonds, which are parts of N-H-I bond configurations except $\mathrm{EAI}^{-1}$. It is worthwhile to note that the atomic vibration shows different dependence based on their polar functional groups. The C-N bond change is the main route of the energy dissipation for $\mathrm{FAI}^{-1}$ and $\mathrm{GAI}^{-1}$. However, the negligible variation can be found for $\mathrm{MAI}^{-1}$ and $\mathrm{EAI}^{-1}$, especially in terms of the initial fluctuation. The N-H bonds show oscillating behaviors for $\mathrm{MAI}^{-1}$ and $\mathrm{EAI}^{-1}$, but rapid damping motion can be found for $\mathrm{FAI}^{-1}$ and $\mathrm{EAI}^{-1}$. The other point is that the magnitude of the bond-length vibration is sensitive to the total molecular mass. The N-H bond vibrations of $\mathrm{MAI}^{-1}$ and $\mathrm{FAI}^{-1}$ are more prominent than those of $\mathrm{EAI}^{-1}$ and $\mathrm{GAI}^{-1}$.

The N-H bond vibration dynamics can elucidate a potential origin of the lattice instability in halide perovskites. It has been commonly accepted that the kinetic path of intrinsic degradation of $\mathrm{MAPbI}_{3}$ starts from the deprotonation of methylammonium cation ${ }^{44,45}$. The strong and localized $\mathrm{N}-\mathrm{H}$ bond vibrational mode of $\mathrm{MAI}^{-1}$ may induce the bond breaking and dissociate $\mathrm{CH}_{3} \mathrm{NH}_{3} \mathrm{I}$ into $\mathrm{CH}_{3} \mathrm{NH}_{2}+\mathrm{HI}$. Although the bond breaking occurs in reality, it is intractable to directly simulate this dissociation process because the pertinent reaction barrier is too high to overcome with a simple electron attachment process. To computationally reproduce this rare event with attosecond resolution, it is necessary to deal with a vast number of samples with different initial ionic kinetic energies for a very long time. Therefore, we will discuss dynamic evidence differentiating the molecular dissociation process instead of directly simulating the event. 

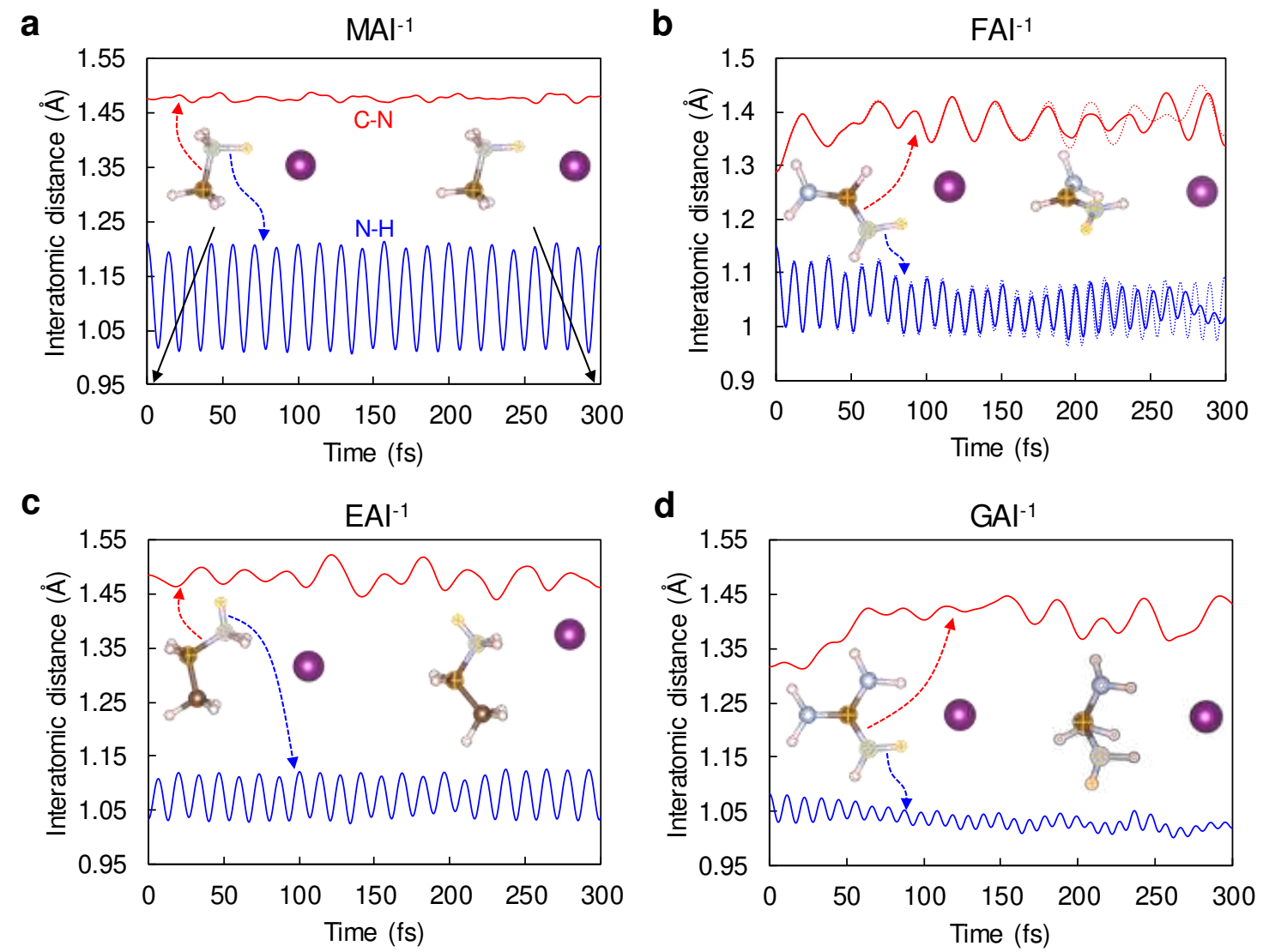

Fig. 2 Time evolution of an N-H distance and a C-N distance in a $\mathrm{MAI}^{-1}, \mathbf{b} \mathrm{FAI}^{-1}, \mathbf{c} \mathrm{EAI}^{-1}$, and $\mathbf{d}$ $\mathrm{GAI}^{-1}$. The inset geometries are snapshots at 0 and $300 \mathrm{fs}$, where corresponding $\mathrm{H}, \mathrm{N}$, and $\mathrm{C}$ are marked by the cross. We select and plot the distances of N-H bonds changing the most for each molecule (see Fig. S3 for interatomic distances between the other N and H). (b) Dotted lines indicate results using $\mathrm{BO}$ approximation, where $0.05 \mathrm{fs}$ was chosen for the time step after the convergence test (Fig. S4).

To grasp the origin of different energy relaxation mechanisms for different cations, the projected density of states (PDOS) and wavefunctions of the anion HOMO state are shown in Fig. 3. Note that the anion HOMO states of the A-I molecules with an additional electron (that is the chemical 
state after the photoexcitation) corresponds to the LUMO of the A-site cation (that is the chemical state before the photoexcitation).

Depending on the chemical moieties, LUMOs of A-site cations show distinct characteristics. For MA and EA belonging to the AMG, the LUMO has a significant contribution from the I $5 p$ orbital (Fig. 3a,c and Fig. S5a,c). This originates from the strong interaction between the A-site cation and I, namely a hydrogen bond that is formed by stabilizing the $\sigma^{*}$ antibonding orbital of the hydrogen bond donor. Comparing anion HOMOs of excited states (Fig. 3a,c) with LUMOs of ground states (Fig. S5a,c), it turns out that this character survives even after electron attachment. Consequently, the strong intermolecular hydrogen bond stabilizes a particular N-H bond. Furthermore, the C-N bond is hardly influenced by the electron attachment according to the LUMO character; thus, the overall molecular structure remains intact. As a result, the stretching vibration of N-H survives for a long time by preventing a fast intramolecular vibrational energy redistribution (IVR), and a fast bond dissociation is eventually expected following the non-RiceRamsperger-Kassel-Marcus (non-RRKM) dynamics ${ }^{46,47}$.

For the A-site cations belonging to the ADG, FA has a LUMO mostly localized on the $\pi^{*}$ antibonding orbital of the ADG (Fig. S5b). This is observed in the anion HOMO of the $\mathrm{FAI}^{-1}$ as well (Fig. 3b). For GA, its LUMO calculated using the zero-kelvin structure of GAI shown in Fig. S5 looks different from the $\pi^{*}$ antibonding orbital although it exhibits originally the $\pi^{*}$ antibonding orbital without I (see Fig. S6 for LUMOs of A-site cations without I). During dynamics after the electron attachment, the anion $\mathrm{HOMO}$ of $\mathrm{GAI}^{-1}$ is converted into its original character, the $\pi^{*}$ antibonding orbital of the ADG, within 50 fs (Fig. S7). In contrast, the conversion does not occur without the additional electron (Fig. S8). Thus, the electron attachment to ADG- 
containing cations makes the $\mathrm{C}-\mathrm{N} \pi$-bond flexible, which opens up new vibrational mixing channels by enabling intramolecular rotation and bending vibration ${ }^{48-50}$. Consequently, a rapid IVR following the conventional RRKM theory is allowed for FA and GA.

a

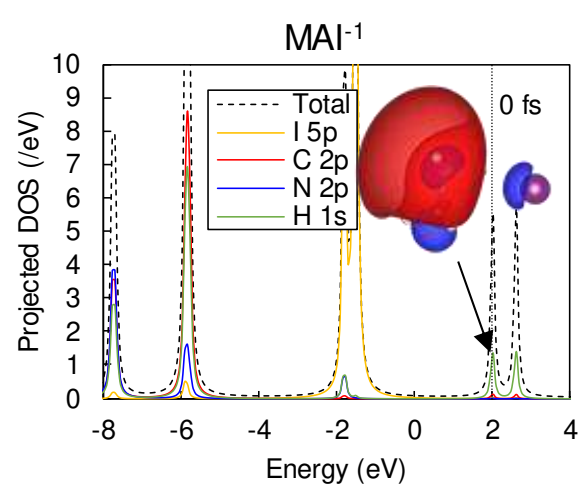

C

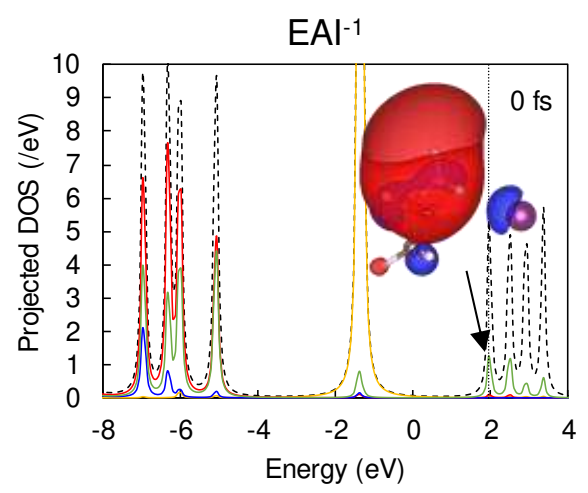

b

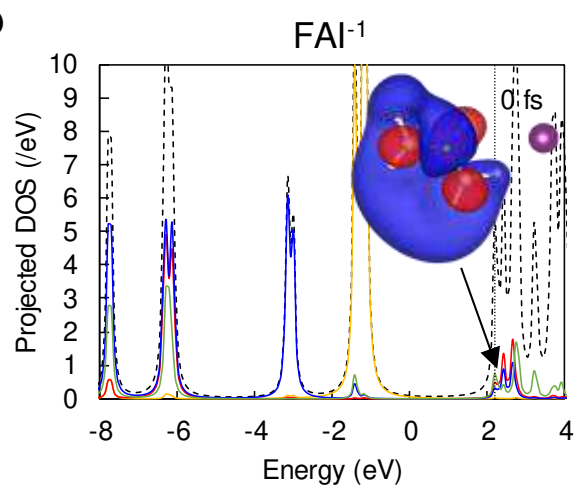

d

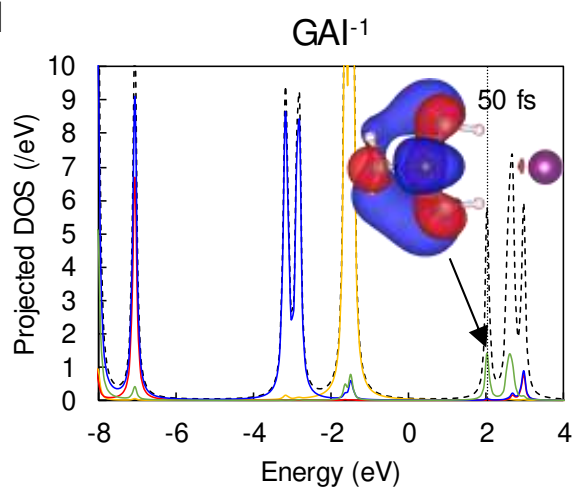

Fig. 3 PDOS of $\mathbf{a ~ M A I}{ }^{-1}, \mathbf{b ~ F A I}{ }^{-1}, \mathbf{c} \mathrm{EAI}^{-1}$, and $\mathbf{d ~ G A I}{ }^{-1}$ at 0 fs. Insets are real parts of wavefunctions of the anion HOMO at given times during dynamics (isosurface level $=0.06 \AA^{-3 / 2}$ ). The initial wavefunction of $\mathrm{GAI}^{-1}$, which is not associated with the $\pi^{*}$ antibonding orbital of the GA, evolves to the $\pi^{*}$ antibonding orbital within $50 \mathrm{fs}$. The vertical dotted lines indicate the Fermi levels. 
The different energy dissipation from carrier excitation can be the source of distinct electronionic coupling behaviors. Figure S9 presents the ion temperature variation for considered A-I molecules during simulation time. The ion temperature of ADG increases significantly faster and higher than that of AMG, which can be attributed to the aforementioned the $\pi^{*}$ antibonding state of C-N bonds of ADG. Our calculation results agree with the previous experimental report, in which FA-based perovskites show faster HC cooling than MA-based or Cs-based one ${ }^{31}$.

Quantum ergodicity of MA and FA. This modeling study found that quantum ergodicity provides an insight into the distinct dynamics of A-site molecules in the two different groups. In the remaining of this paper, we discuss a consequence of non-adiabatic dynamics; then the quantum ergodicity of MA and FA is investigated in terms of energy level spectra.

We note that the TDDFT-based Ehrenfest dynamics result in different dynamics from the BornOppenheimer (BO) approximation owing to the limitation of adiabatic dynamics in describing the electron attached systems (Fig. 2b). Non-adiabatic dynamics can appear when the initial forces on ions are nonzero even if dynamics begin with the electronic ground state ${ }^{51}$. Further analysis of orbital occupation as a function of time provides additional information about the deviation from the $\mathrm{BO}$ approximation and the fast IVR in $\mathrm{MAI}^{-1}$ and $\mathrm{FAI}^{-1}$ (Fig. 4). These populations are obtained by projecting time-evolving Kohn-Sham (KS) orbitals into KS eigenstates, which are calculated using diagonalization with respect to the ion geometry and electron density at a given time. See Fig. S10 for other physical quantities, such as ion temperature, ion-ion potential energy, electron energy, and KS eigenvalues as functions of time. There are two distinct modulations in the occupation around $0 \mathrm{fs}$ and $100 \mathrm{fs}$. At $0 \mathrm{fs}$, an electron in the 14th spin-up orbital (anion HOMO) of $\mathrm{FAI}^{-1}$ is excited into the 15 th orbital. However, it hardly affects the molecular dynamics because the orbital character of the anion HOMO resembles that of the higher one, where they are originally 
degenerate in the neutral charge state. On the other hand, around $100 \mathrm{fs}$, an electron in the 13th spin-down orbital is excited into the 14th orbital through the non-adiabatic dynamics, and this accounts for the discrepancy between the Ehrenfest dynamics and the BO approximation (Fig. 2b). Occupying the 14 th spin-down orbital, which still possesses the $\pi^{*}$ antibonding character albeit distorted, imposes anharmonic vibrational coupling, which facilitates $\mathrm{IVR}^{37}$. One may argue that the amount of the population change at 100 fs is only 0.005 ; the small occupation of the 14 th orbital, however, can lead to a drastic difference in a later time in the sense of chaos. On the contrary to the $\mathrm{FAI}^{-1}$, the occupation in $\mathrm{MAI}^{-1}$ does not change in time along with long-lived local vibration modes shown in Fig. 2a

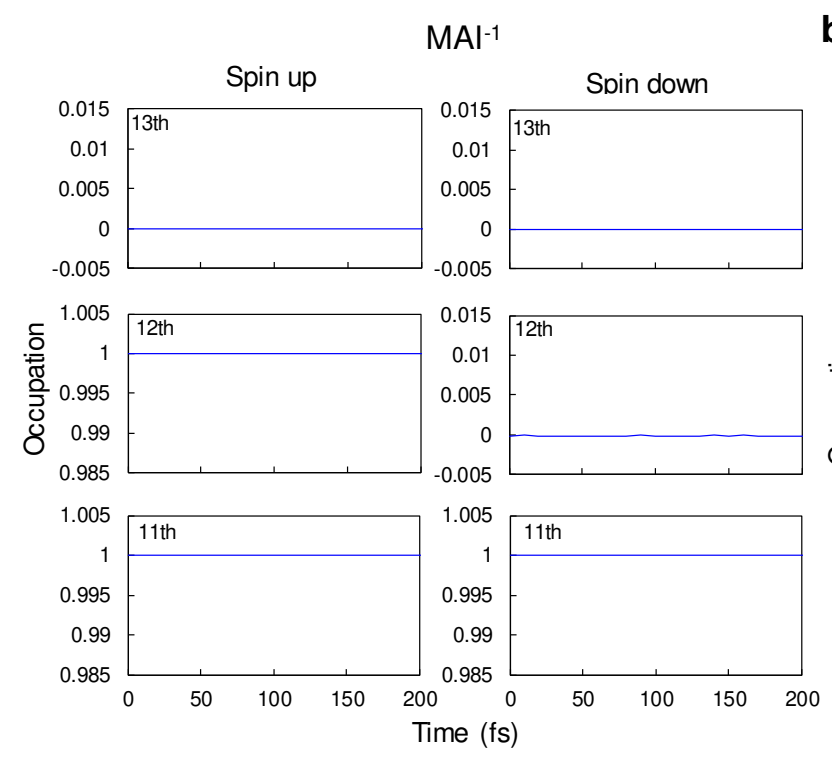

b
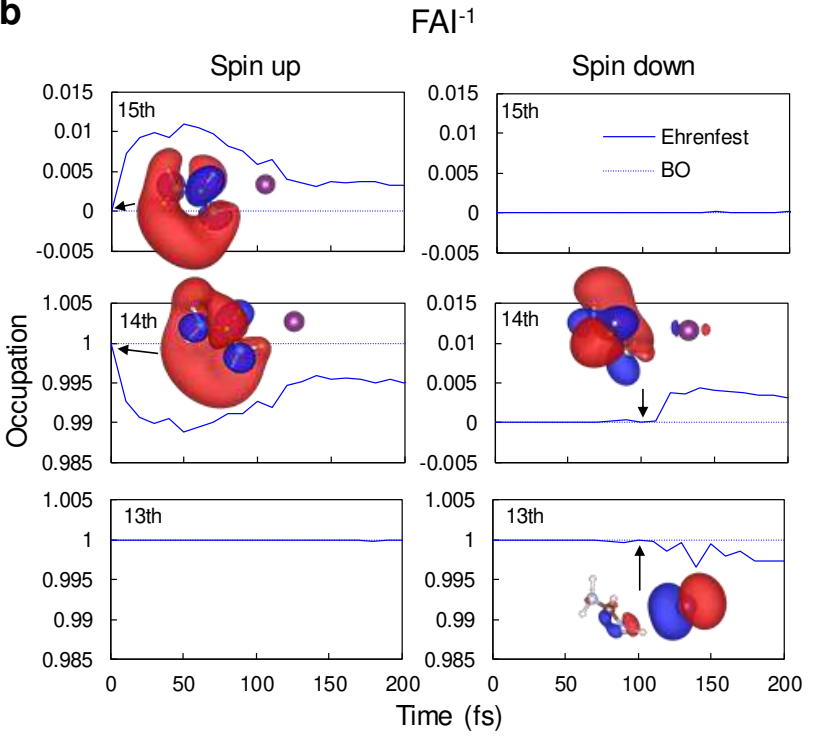

Fig. 4 Time-dependent occupation of KS orbitals. a Occupation of KS orbitals of MAI ${ }^{-1}$. The 12th spin-up orbital corresponds to the anion HOMO of $\mathrm{MAI}^{-1}$. Despite the non-adiabatic simulation, the occupation does not change as time passes. b Occupation of KS orbitals of $\mathrm{FAI}^{-1}$. The 14th 
spin-up orbital is the anion HOMO. Insets are the real parts of corresponding wavefunctions of the 14th and 15th spin-up states at $0 \mathrm{fs}$ and the 13th and 14th spin-down states at $100 \mathrm{fs}$.

As aforementioned, slow IVR associated with non-RRKM dynamics is responsible for fast unimolecular dissociation due to non-ergodic behavior ${ }^{47}$. In this regard, the slow IVR in the AMG likely accounts for fast hydrogen dissociation followed by photodegradation of perovskite solar cells $^{27}$, and relatively stable perovskite solar cells have been fabricated by incorporating the $\mathrm{ADG}^{20-22}$. Interestingly, the fast IVR in ADG can be interpreted in terms of chaotic eigenvalue dynamics (see Fig. S11 and Fig. S12 for the time evolution of KS eigenvalues of $\mathrm{MAI}^{-1}$ and $\mathrm{FAI}^{-}$ $\left.{ }^{1}\right)$. According to the random matrix theory (RMT), a chaotic system is characterized by the Gaussian orthogonal ensemble (GOE), in which the nearest-neighbor level-spacing distribution belongs to Wigner-Dyson statistics; whereas a Poisson distribution is expected when eigenvalues are uncorrelated ${ }^{38,39}$. On top of that, the level-spacing distribution has been investigated in molecular systems to see energy level correlations and how chaotic they $\operatorname{are}^{52-54}$.

Taking into account time-evolving KS eigenvalues, we calculate level-spacing distributions of $\mathrm{MAI}^{-1}$ and $\mathrm{FAI}^{-1}$ as a function of time, which shows the different IVR processes between considered functional groups (Fig. 5). It turns out that while the level spacings of $\mathrm{MAI}^{-1}$ keep belonging to the Poisson distribution for 300 fs (see Fig. S13 for long-time dynamics), those of FAI $^{-1}$ rapidly change from the Poisson distribution to the Wigner-Dyson distribution within tens of femtosecond. The Wigner-Dyson statistics is a manifestation of more chaotic dynamics of the corresponding system, which has to do with quantum ergodicity responsible for faster IVR in $\mathrm{FAI}^{-}$ ${ }^{1}$. Another way of interpreting the fast IVR in $\mathrm{FAI}^{-1}$ with the level-spacing distribution is that the 
Wigner-Dyson statistics in $\mathrm{FAI}^{-1}$ is a consequence of level repulsion induced by symmetry breaking, and the IVR process is faster in the molecules of lower symmetry ${ }^{55}$. Accordingly, the electronic quantum chaos turns out to play an essential role in the ergodicity of ion dynamics, responsible for the fast IVR.

a
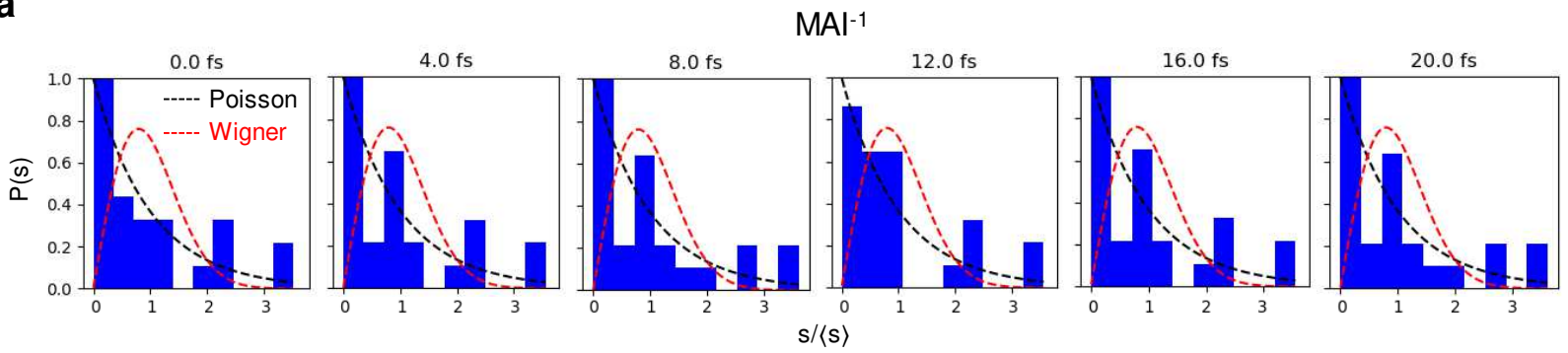

b
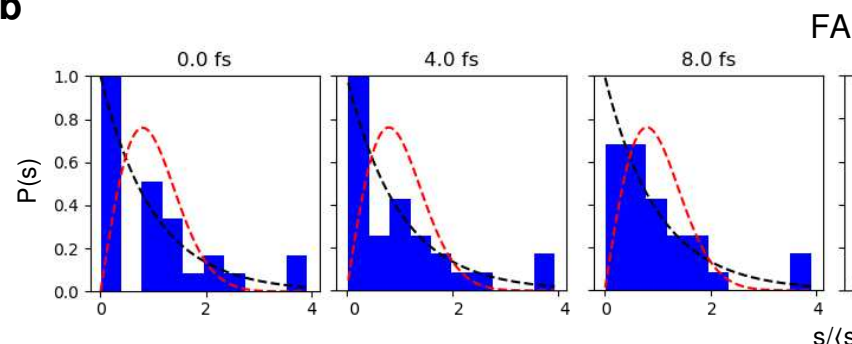

$\mathrm{FAl}^{-1}$
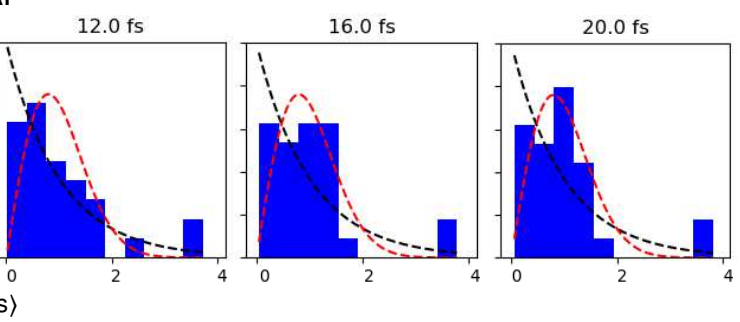

Fig. 5 Level-spacing distribution of KS eigenvalues from 0 to 20 fs in $\mathbf{a} \mathrm{MAI}^{-1}$ and $\mathbf{b} \mathrm{FAI}^{-1}$. In $\mathrm{FAI}^{-1}$, the distribution rapidly changes from Poisson to Wigner-Dyson statistics within $20 \mathrm{fs}$. A Poisson distribution is described in $P(s)=\frac{1}{\langle s\rangle} e^{-s /\langle s\rangle}$, and a Wigner-Dyson distribution is described in $P(s)=\frac{\pi s}{2\langle s\rangle^{2}} e^{-\left(\pi s^{2} / 4\langle s\rangle^{2}\right)}$, where $\langle s\rangle$ is the mean spacing between adjacent levels. 


\section{Discussion}

In summary, we show that the role of molecular cations on the femtosecond quantum dynamics of excited states using TDDFT calculations with essential non-adiabatic features. The calculation results present that A-site cation molecules can have significant impacts on the stability and the carrier dynamics during the thermalization of excited carriers. The energy dissipation processes of excited A-site cations are determined by the chemical bonding, especially the LUMO state of Asite cations. The LUMO of the ADG is made of the $\sigma^{*}$ antibonding state of the hydrogen bonding with iodine in inorganic lattices. When the LUMO is occupied with an excitation, a substantial, long-lived N-H vibration induces deprotonation readily (non-RRKM). On the contrary, since the LUMO consisting of $\pi^{*}$ antibonding state within a molecule in the AMG makes the molecule chaotic, so that FA (or GA) can facilitate dissipation of the excitation energy with the IVR, resulting in slow molecular dissociation (RRKM).

This study also presents that the energy dissipation process from excited states of A-site cation molecules is closely relevant to the quantum chaos and ergodicity. The energy level spectrum analysis based on quantum chaos reveals that the electronic quantum chaos of ADG plays an essential role in the ergodicity of ion dynamics, responsible for the fast IVR.

This work suggests the theoretical background of the reason why FA-based perovskites can have better photostability than MA-based ones. Our in-depth analysis presents that quantum ergodicity is the descriptor for the intrinsic photostability of organic-inorganic hybrid perovskites. Moreover, our calculation enlightens the importance of the chemical bonding nature of A-site molecule for the stability and carrier dynamics under the light irradiation. 


\section{Methods}

TDDFT modeling. The real-time TDDFT ${ }^{35,36,56,57}$ in the framework of Ehrenfest dynamics ${ }^{58,59}$ is employed to carry out non-adiabatic ab initio molecular dynamics study. The approach has been implemented in the real-space code, Octopus ${ }^{60-63}$. We use the set of the Hartwigsen-GoedeckerHutter $(\mathrm{HGH})^{64}$ pseudopotentials for local density approximation (LDA) ${ }^{65}$, and the adiabatic approximation $^{36}$ is employed for the exchange-correlation (XC) functional in TDDFT. The spacing between the points in the real-space mesh is set to $0.18 \AA$, and the time step $\Delta t$ for integration is set to $0.0023 \mathrm{~h} / \mathrm{eV}$. We have confirmed the total energy conservation for simulation time to verify computational stability and accuracy. Coupled electron-ion dynamics driven by electron attachment is simulated as follows. First, with a neutral charge, geometry is optimized until the maximum force is smaller than $0.05 \mathrm{eV} / \AA$. Then, we manipulate occupation numbers of orbitals to add an electron into the lowest unoccupied molecular orbital (LUMO) of the original system, which then turns into the highest occupied molecular orbital (HOMO) of the modified system. This is referred to as anion HOMO. Once electronic minimization is performed with the original geometry, initial forces are exerted on ions. Finally, we let the system evolve in time with the initial forces based on the TDDFT-based Ehrenfest dynamics.

\section{Acknowledgments}

This work was supported by ASCENT, one of six centers in JUMP, a Semiconductor Research Corporation (SRC) program sponsored by DARPA (Grant No. 2018-JU-2776). This work was 
also supported by Creative Materials Discovery Program (Grant No. 2015M3D1A1068062) and Nano Materials Technology Development Program (Grant No. 2016M3A7B4909942) through the National Research Foundation of Korea (NRF) funded by Ministry of Science and ICT. K.-H.H. acknowledges financial supports by Hanbat National University Financial Accounting Research Fund (2019 year), the National R\&D Program through the Korean NRF (Grant Nos. NRF2015M1A2A2055836, NRF-2018R1A2B6007888, NRF-2017M3A7B4041698), and New \&

Renewable Energy Core Technology Program of the Korea Institute of Energy Technology Evaluation and Planning (KETEP) (Grant No. 20183010013820). We acknowledge the Texas Advanced Computing Center (TACC) for providing supercomputing resources.

\section{Author contributions}

Y.L. performed the calculation and analyzed the data; All authors (Y.L., H.K., K-.H.H., and K.C.) contributed to idea development, discussion, and preparation of the manuscript.

\section{Additional information}

Supplementary Information accompanies this paper at https://.

\section{References}

1. Jung, E. H. et al. Efficient, stable and scalable perovskite solar cells using poly(3hexylthiophene). Nature 567, 511-515 (2019).

2. Lee, J. \& Park, N. Chemical Approaches for Stabilizing Perovskite Solar Cells. Adv. Energy Mater. 10, 1903249 (2020). 
3. Chen, J., Zhao, X., Kim, S. \& Park, N. Multifunctional Chemical Linker Imidazoleacetic Acid Hydrochloride for 21\% Efficient and Stable Planar Perovskite Solar Cells. Adv. Mater. 31, 1902902 (2019).

4. Jiang, Q. et al. Surface passivation of perovskite film for efficient solar cells. Nat. Photonics 13, 460-466 (2019).

5. Wadi, M. A. A. et al. Evolution of Pb-Free and Partially Pb-Substituted Perovskite Absorbers for Efficient Perovskite Solar Cells. Electron. Mater. Lett. 15, 525-546 (2019).

6. Lin, K. et al. Perovskite light-emitting diodes with external quantum efficiency exceeding 20 per cent. Nature 562, 245-248 (2018).

7. Tan, Z.-K. et al. Bright light-emitting diodes based on organometal halide perovskite. Nat. Nanotechnol. 9, 687-692 (2014).

8. Quan, L. N. et al. Perovskites for Next-Generation Optical Sources. Chem. Rev. 119, 7444 7477 (2019).

9. Hwang, B., Gu, C., Lee, D. \& Lee, J.-S. Effect of halide-mixing on the switching behaviors of organic-inorganic hybrid perovskite memory. Sci. Rep. 7, 43794 (2017).

10. Choi, J. et al. Organolead Halide Perovskites for Low Operating Voltage Multilevel Resistive Switching. Adv. Mater. 28, 6562-6567 (2016).

11. Kang, D.-H. et al. An Ultrahigh-Performance Photodetector based on a PerovskiteTransition-Metal-Dichalcogenide Hybrid Structure. Adv. Mater. 28, 7799-7806 (2016).

12. Wang, H. \& Kim, D. H. Perovskite-based photodetectors: materials and devices. Chem. Soc. Rev. 46, 5204-5236 (2017).

13. Cao, Y. et al. Perovskite light-emitting diodes based on spontaneously formed submicrometre-scale structures. Nature 562, 249-253 (2018).

14. Kojima, A., Teshima, K., Shirai, Y. \& Miyasaka, T. Organometal Halide Perovskites as Visible-Light Sensitizers for Photovoltaic Cells. J. Am. Chem. Soc. 131, 6050-6051 (2009).

15. Eperon, G. E. et al. Formamidinium lead trihalide: a broadly tunable perovskite for efficient planar heterojunction solar cells. Energy Environ. Sci. 7, 982 (2014).

16. Im, J.-H., Chung, J., Kim, S.-J. \& Park, N.-G. Synthesis, structure, and photovoltaic property of a nanocrystalline $2 \mathrm{H}$ perovskite-type novel sensitizer $\left(\mathrm{CH}_{3} \mathrm{CH}_{2} \mathrm{NH}_{3}\right) \mathrm{PbI}_{3}$. Nanoscale Res. Lett. 7, 353 (2012).

17. Giorgi, G., Fujisawa, J.-I., Segawa, H. \& Yamashita, K. Organic-Inorganic Hybrid Lead Iodide Perovskite Featuring Zero Dipole Moment Guanidinium Cations: A Theoretical Analysis. J. Phys. Chem. C 119, 4694-4701 (2015).

18. Krishna, A., Gottis, S., Nazeeruddin, M. K. \& Sauvage, F. Mixed Dimensional 2D/3D Hybrid Perovskite Absorbers: The Future of Perovskite Solar Cells? Adv. Funct. Mater. 29, 1806482 (2019). 
19. Grancini, G. \& Nazeeruddin, M. K. Dimensional tailoring of hybrid perovskites for photovoltaics. Nat. Rev. Mater. 4, 4-22 (2019).

20. Lee, J.-W., Seol, D.-J., Cho, A.-N. \& Park, N.-G. High-Efficiency Perovskite Solar Cells Based on the Black Polymorph of $\mathrm{HC}\left(\mathrm{NH}_{2}\right)_{2} \mathrm{PbI}_{3}$. Adv. Mater. 26, 4991-4998 (2014).

21. Yamamoto, K. et al. Degradation mechanism for planar heterojunction perovskite solar cells. Jpn. J. Appl. Phys. 55, 04ES07 (2016).

22. Lee, J.-W. et al. Formamidinium and Cesium Hybridization for Photo- and MoistureStable Perovskite Solar Cell. Adv. Energy Mater. 5, 1501310 (2015).

23. Frost, J. M. et al. Atomistic Origins of High-Performance in Hybrid Halide Perovskite Solar Cells. Nano Lett. 14, 2584-2590 (2014).

24. Jodlowski, A. D. et al. Large guanidinium cation mixed with methylammonium in lead iodide perovskites for 19\% efficient solar cells. Nat. Energy 2, 972-979 (2017).

25. Peng, W. et al. Engineering of $\mathrm{CH}_{3} \mathrm{NH}_{3} \mathrm{PbI}_{3}$ Perovskite Crystals by Alloying Large Organic Cations for Enhanced Thermal Stability and Transport Properties. Angew. Chem. Int. Ed. 55, 10686-10690 (2016).

26. Lang, F. et al. Influence of Radiation on the Properties and the Stability of Hybrid Perovskites. Adv. Mater. 30, 1702905 (2018).

27. Nickel, N. H., Lang, F., Brus, V. V., Shargaieva, O. \& Rappich, J. Unraveling the LightInduced Degradation Mechanisms of $\mathrm{CH}_{3} \mathrm{NH}_{3} \mathrm{PbI}_{3}$ Perovskite Films. Adv. Electron. Mater. 3, 1700158 (2017).

28. Song, Z. et al. Probing the origins of photodegradation in organic-inorganic metal halide perovskites with time-resolved mass spectrometry. Sustain. Energy Fuels 2, 2460-2467 (2018).

29. Hong, Q.-M., Xu, R.-P., Jin, T.-Y., Tang, J.-X. \& Li, Y.-Q. Unraveling the light-induced degradation mechanism of $\mathrm{CH}_{3} \mathrm{NH}_{3} \mathrm{PbI}_{3}$ perovskite films. Organ. Electron. 67, 19-25 (2019).

30. Fang, H.-H. et al. Unravelling Light-Induced Degradation of Layered Perovskite Crystals and Design of Efficient Encapsulation for Improved Photostability. Adv. Funct. Mater. 28, 1800305 (2018).

31. Chen, J., Messing, M. E., Zheng, K. \& Pullerits, T. Cation-Dependent Hot Carrier Cooling in Halide Perovskite Nanocrystals. J. Am. Chem. Soc. 141, 3532-3540 (2019).

32. Ross, R. T. \& Nozik, A. J. Efficiency of hot-carrier solar energy converters. J. Appl. Phys. 53, 3813-3818 (1982).

33. Liu, J. \& Prezhdo, O. V. Chlorine Doping Reduces Electron-Hole Recombination in Lead Iodide Perovskites: Time-Domain Ab Initio Analysis. J. Phys. Chem. Lett. 6, 4463-4469 (2015).

34. He, J., Fang, W.-H., Long, R. \& Prezhdo, O. V. Increased Lattice Stiffness Suppresses Nonradiative Charge Recombination in $\mathrm{MAPbI}_{3}$ Doped with Larger Cations: Time-Domain $\mathrm{Ab}$ Initio Analysis. ACS Energy Lett. 3, 2070-2076 (2018). 
35. Runge, E. \& Gross, E. K. U. Density-Functional Theory for Time-Dependent Systems. Phys. Rev. Lett. 52, 997-1000 (1984).

36. Maitra, N. T. Perspective: Fundamental aspects of time-dependent density functional theory. J. Chem. Phys. 144, 220901 (2016).

37. Stechel, E. B. \& Heller, E. J. Quantum Ergodicity and Spectral Chaos. Annu. Rev. Phys. Chem. 35, 563-589 (1984).

38. Guhr, T., Müller-Groeling, A. \& Weidenmüller, H. A. Random-matrix theories in quantum physics: common concepts. Phys. Rep. 299, 189-425 (1998).

39. Stöckmann, H.-J. Quantum chaos: an introduction. (Cambridge Univ. Press, 2006).

40. Leitner, D. M. Quantum ergodicity and energy flow in molecules. Adv. Phys. 64, 445-517 (2015).

41. Kasha, M. Characterization of electronic transitions in complex molecules. Discuss. Faraday Soc. 9, 14 (1950).

42. Agiorgousis, M. L., Sun, Y.-Y., Zeng, H. \& Zhang, S. Strong Covalency-Induced Recombination Centers in Perovskite Solar Cell Material $\mathrm{CH}_{3} \mathrm{NH}_{3} \mathrm{PbI}_{3}$. J. Am. Chem. Soc. 136, 14570-14575 (2014).

43. Meggiolaro, D. et al. Iodine chemistry determines the defect tolerance of lead-halide perovskites. Energy Environ. Sci. 11, 702-713 (2018).

44. Thampy, S., Zhang, B., Hong, K.-H., Cho, K. \& Hsu, J. W. P. Altered Stability and Degradation Pathway of $\mathrm{CH}_{3} \mathrm{NH}_{3} \mathrm{PbI}_{3}$ in Contact with Metal Oxide. ACS Energy Lett. 5, 11471152 (2020).

45. Ciccioli, A. \& Latini, A. Thermodynamics and the Intrinsic Stability of Lead Halide Perovskites $\mathrm{CH}_{3} \mathrm{NH}_{3} \mathrm{PbX}_{3}$. J. Phys. Chem. Lett. 9, 3756-3765 (2018).

46. Madjet, M. E. et al. Cation Effect on Hot Carrier Cooling in Halide Perovskite Materials. J. Phys. Chem. Lett. 8, 4439-4445 (2017).

47. Diau, E. W. Femtosecond Activation of Reactions and the Concept of Nonergodic Molecules. Science 279, 847-851 (1998).

48. Dai, H. L., Korpa, C. L., Kinsey, J. L. \& Field, R. W. Rotation-induced vibrational mixing in $X^{1} A_{1}$ formaldehyde: Non-negligible dynamical consequences of rotation. J. Chem. Phys. 82, 1688-1701 (1985).

49. Apel, E. C. \& Lee, E. K. C. Rotation-induced vibrational mixing in $S_{1} \mathrm{H}_{2} \mathrm{CO}$ near $E_{\text {vib }}^{\prime}=$ 2000-2300 $\mathrm{cm}^{-1}: 2^{1} 4^{3}$ and $2^{1} 4^{1} 6^{1}$ levels. J. Chem. Phys. 85, 1261-1275 (1986).

50. Bethardy, G. A., Wang, X. \& Perry, D. S. The role of molecular flexibility in accelerating intramolecular vibrational relaxation. Can. J. Chem. 72, 652-659 (1994). 
51. Ojanperä, A., Havu, V., Lehtovaara, L. \& Puska, M. Nonadiabatic Ehrenfest molecular dynamics within the projector augmented-wave method. J. Chem. Phys. 136, 144103 (2012).

52. Zimmermann, Th., Köppel, H., Cederbaum, L. S., Persch, G. \& Demtröder, W. Confirmation of Random-Matrix Fluctuations in Molecular Spectra. Phys. Rev. Lett. 61, 3-6 (1988).

53. Polik, W. F., Guyer, D. R., Miller, W. H. \& Moore, C. B. Eigenstate-resolved unimolecular reaction dynamics: Ergodic character of $S_{0}$ formaldehyde at the dissociation threshold. J. Chem. Phys. 92, 3471-3484 (1990).

54. Leitner, D. M., Köppel, H. \& Cederbaum, L. S. Statistical properties of molecular spectra and molecular dynamics: Analysis of their correspondence in $\mathrm{NO}_{2}$ and $\mathrm{C}_{2} \mathrm{H}_{4}^{+}$. J. Chem. Phys. 104, 434-443 (1996).

55. von Benten, R. S., Liu, Y. \& Abel, B. Dynamical consequences of symmetry breaking in benzene and difluorobenzene. J. Chem. Phys. 133, 134306 (2010).

56. Hohenberg, P. \& Kohn, W. Inhomogeneous Electron Gas. Phys. Rev. 136, B864-B871 (1964).

57. Kohn, W. \& Sham, L. J. Self-Consistent Equations Including Exchange and Correlation Effects. Phys. Rev. 140, A1133-A1138 (1965).

58. Li, X., Tully, J. C., Schlegel, H. B. \& Frisch, M. J. Ab initio Ehrenfest dynamics. J. Chem. Phys. 123, 084106 (2005).

59. Parandekar, P. V. \& Tully, J. C. Mixed quantum-classical equilibrium. J. Chem. Phys. 122, $094102(2005)$.

60. Marques, M. octopus: a first-principles tool for excited electron-ion dynamics. Comput. Phys. Commun. 151, 60-78 (2003).

61. Castro, A. et al. octopus: a tool for the application of time-dependent density functional theory. Phys. status solidi (b) 243, 2465-2488 (2006).

62. Andrade, X. et al. Real-space grids and the Octopus code as tools for the development of new simulation approaches for electronic systems. Phys. Chem. Chem. Phys. 17, 31371-31396 (2015).

63. Tancogne-Dejean, N. et al. Octopus, a computational framework for exploring light-driven phenomena and quantum dynamics in extended and finite systems. J. Chem. Phys. 152, 124119 (2020).

64. Hartwigsen, C., Goedecker, S. \& Hutter, J. Relativistic separable dual-space Gaussian pseudopotentials from H to Rn. Phys. Rev. B 58, 3641-3662 (1998).

65. Parr, R. G. \& Yang, W. Density-functional theory of atoms and molecules. (Oxford Univ. Press [u.a.], 1994). 


\section{Figures}

a
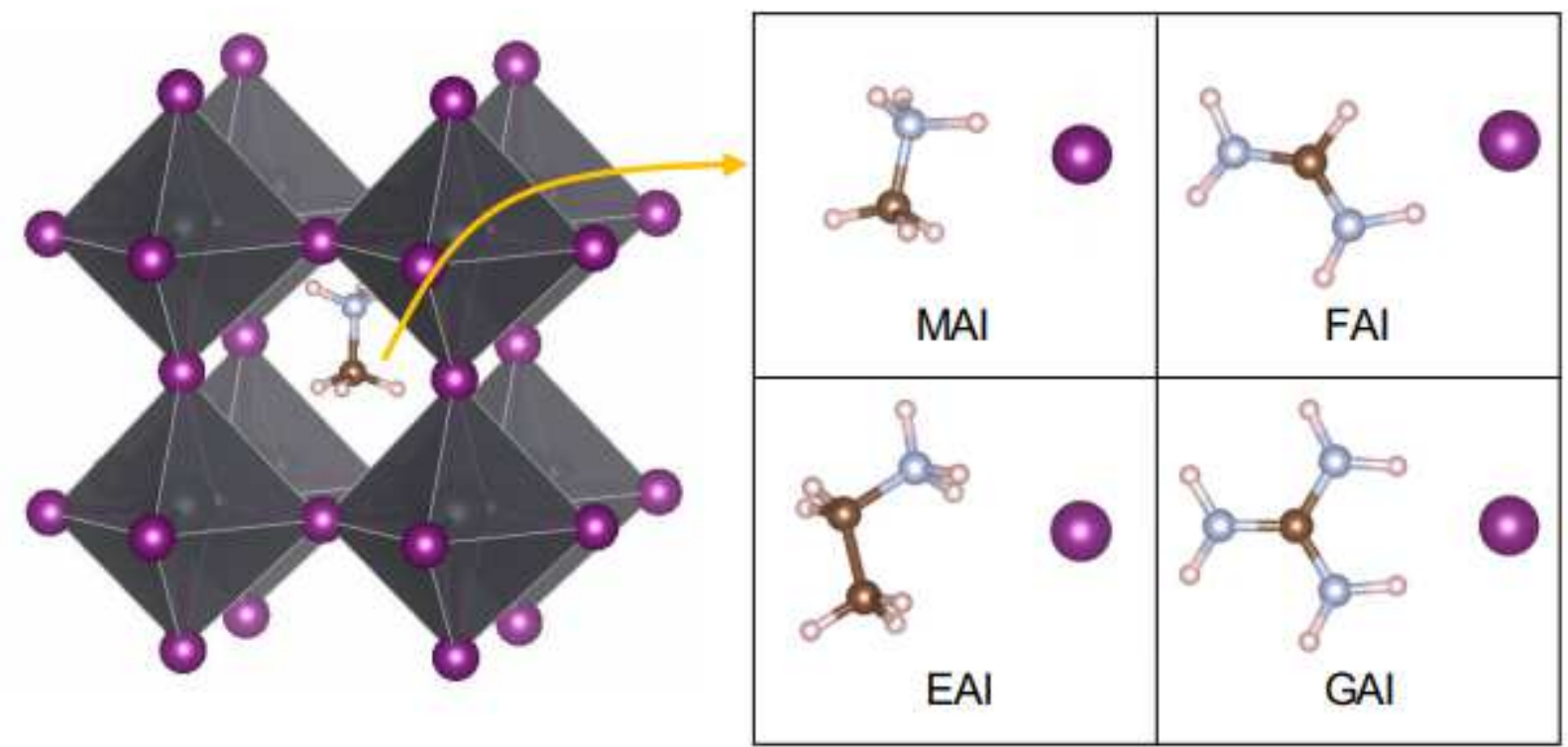

$* \mathrm{~Pb} \odot \mathrm{I} \circ \mathrm{H} \quad \circ \mathrm{N} \quad \odot \mathrm{C}$

b

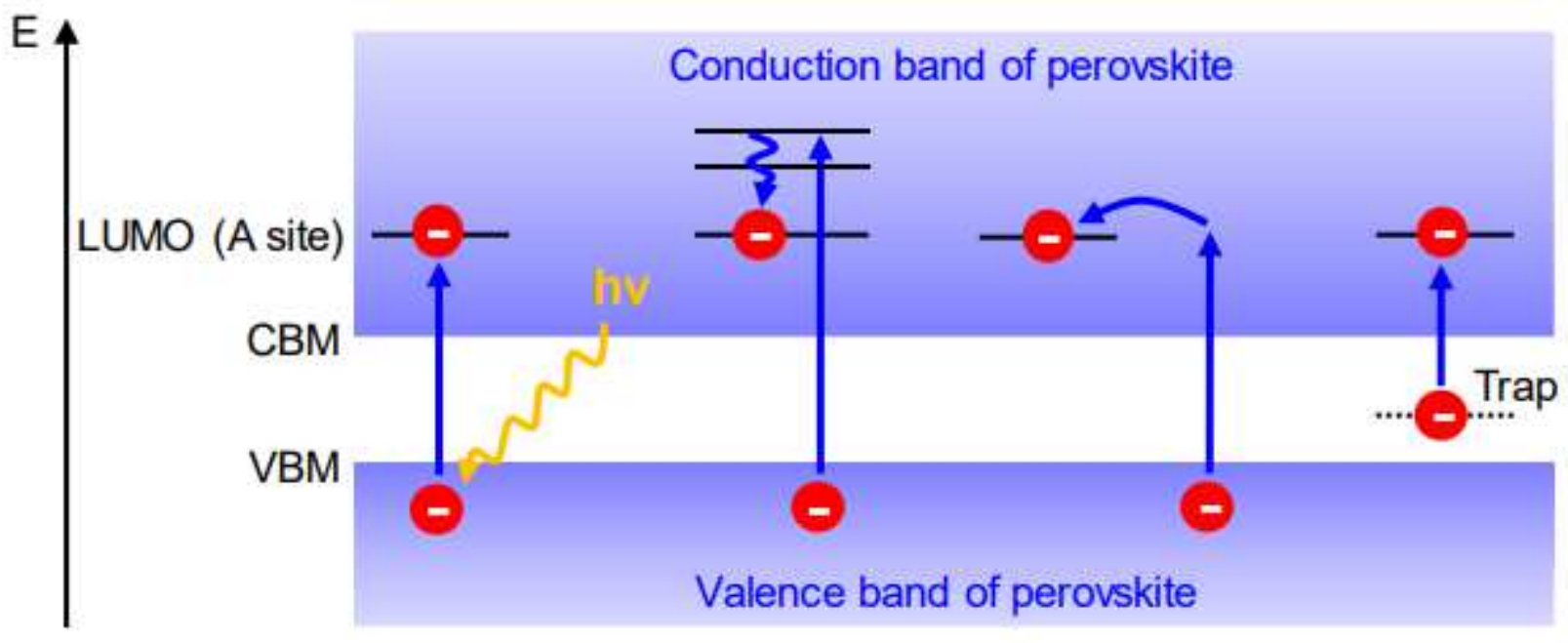

Figure 1

a Atomistic structure of organic-inorganic hybrid perovskites (left). To study the lightinduced dynamics of A-site organic cations in the perovskites, we consider molecular systems of an organic cation attached to an iodine anion (right). b Schematic of electron capture processes into the LUMO of A-site cations. Energy levels of three-dimensional (3D) perovskites and molecules are represented by shaded regions and solid lines, respectively. A dotted line represents a trap state. 
a

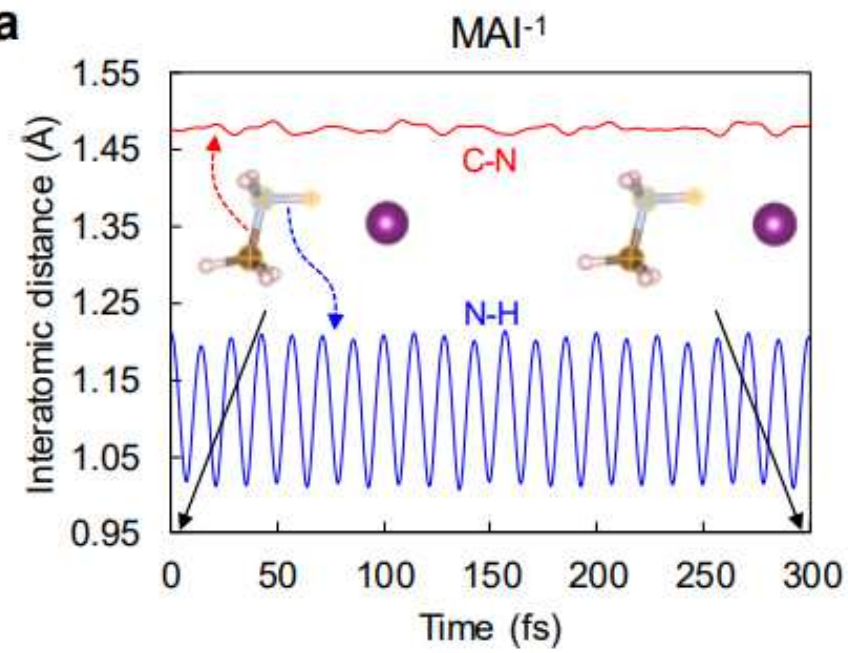

C

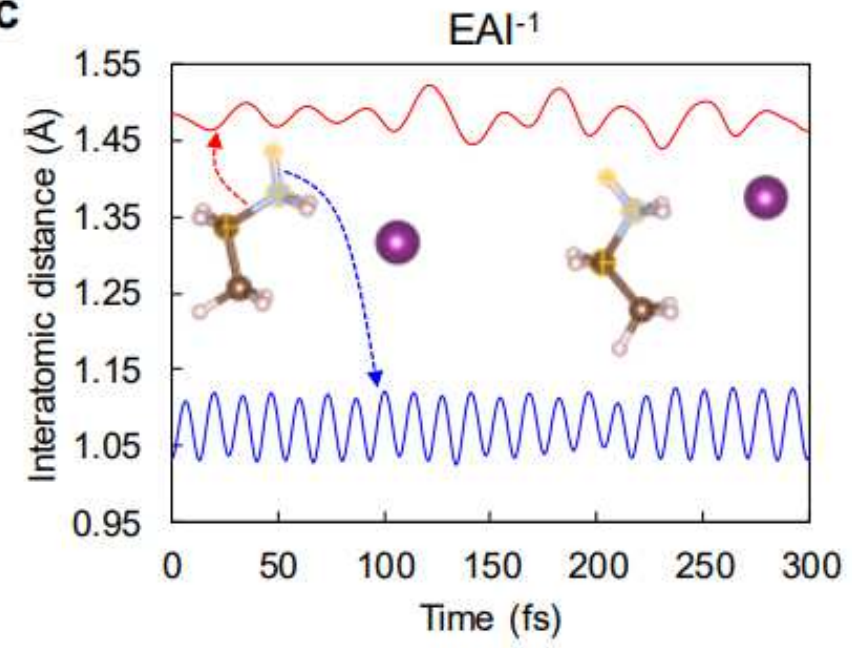

b

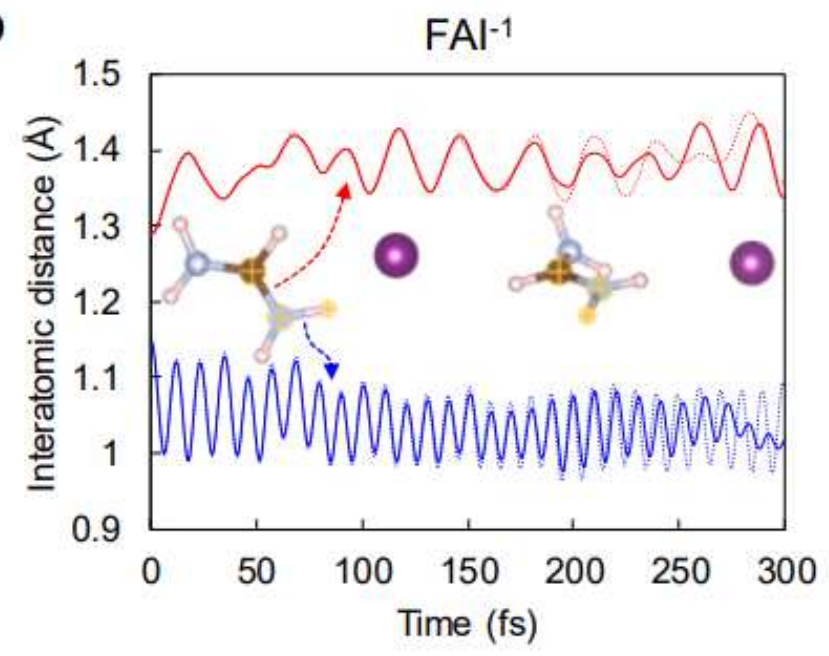

d

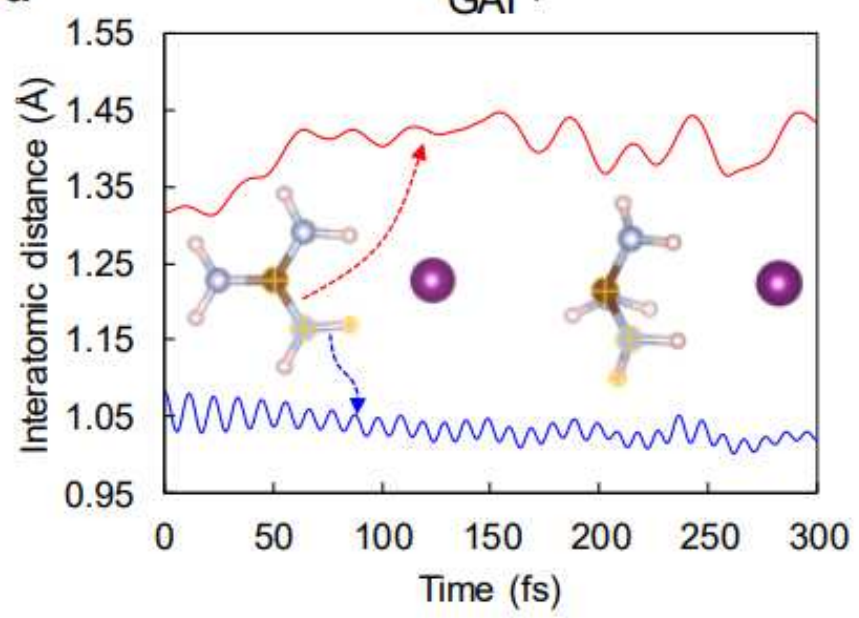

Figure 2

Time evolution of an N-H distance and a C-N distance in a MAl-1 , b FAl-1 , c EAl-1, and d GAl-1. The inset geometries are snapshots at 0 and $300 \mathrm{fs}$, where corresponding $\mathrm{H}, \mathrm{N}$, and $\mathrm{C}$ are marked by the cross. We select and plot the distances of $\mathrm{N}-\mathrm{H}$ bonds changing the most for each molecule (see Fig. S3 for interatomic distances between the other $\mathrm{N}$ and $\mathrm{H}$ ). (b) Dotted lines indicate results using BO approximation, where $0.05 \mathrm{fs}$ was chosen for the time step after the convergence test (Fig. S4). 
a

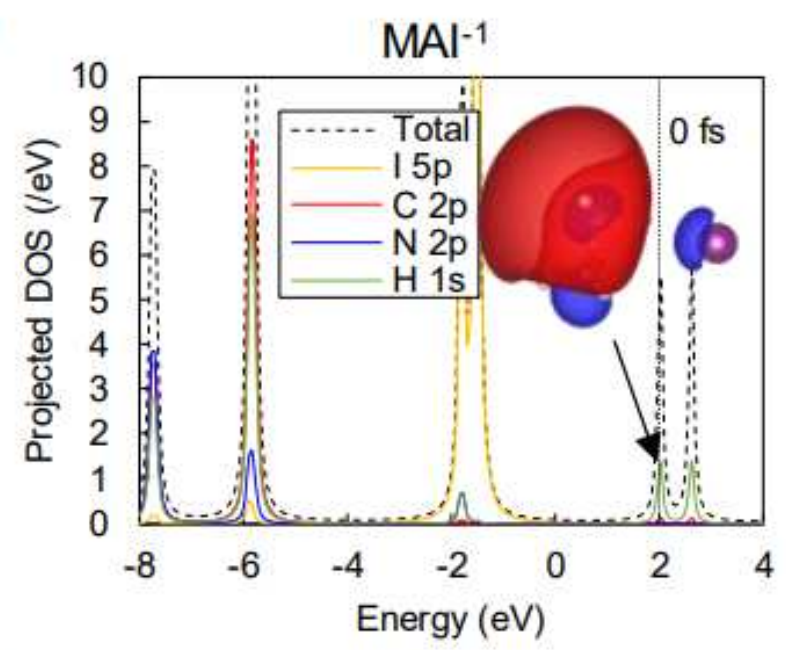

C

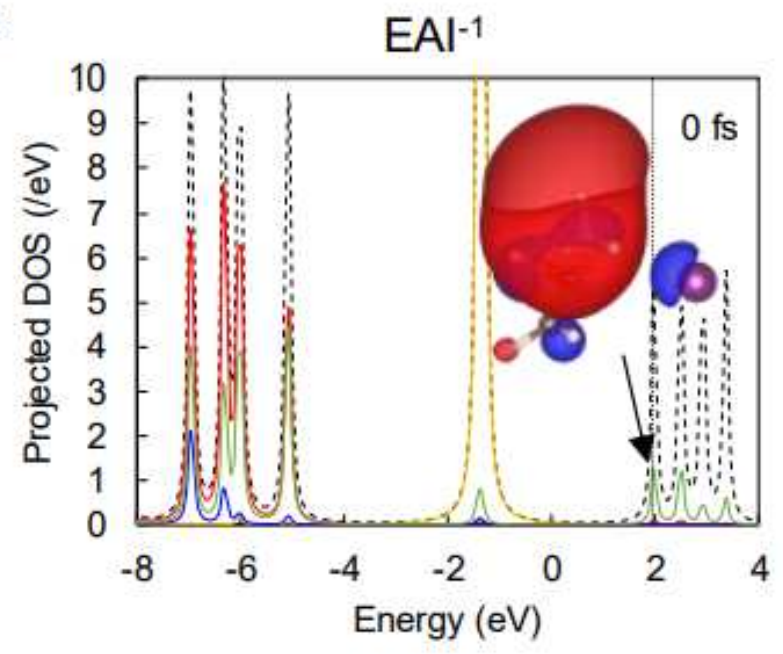

b

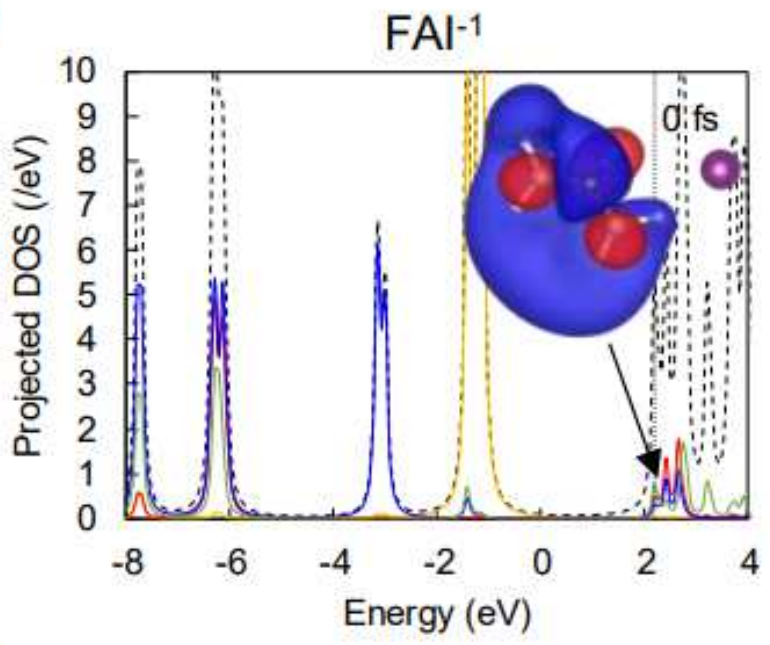

d

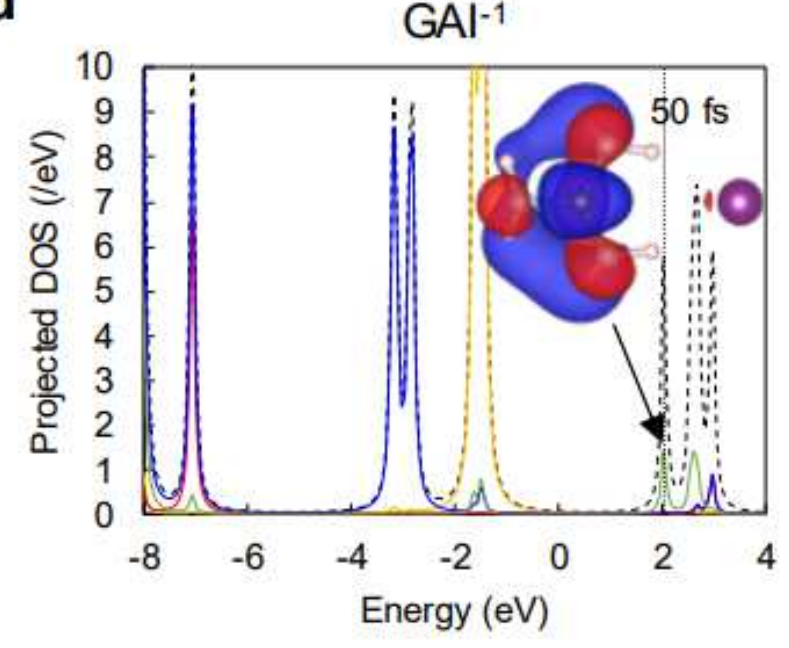

Figure 3

PDOS of a MAl-1 , b FAl-1 , c EAl-1, and d GAl-1 at $0 \mathrm{fs}$. Insets are real parts of wavefunctions of the anion $\mathrm{HOMO}$ at given times during dynamics (isosurface level $=0.06 \AA-3 / 2$ ). The initial wavefunction of GAl-1, which is not associated with the $\pi^{\star}$ antibonding orbital of the GA, evolves to the $\pi^{\star}$ antibonding orbital within $50 \mathrm{fs}$. The vertical dotted lines indicate the Fermi levels. 


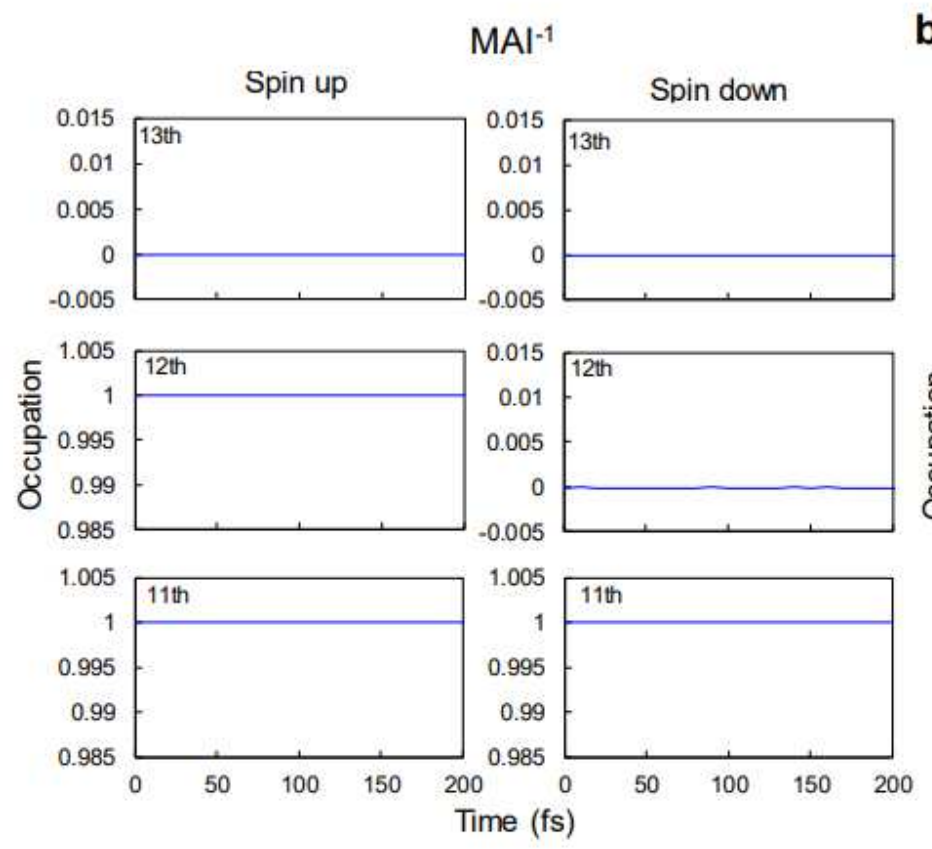

b

$\mathrm{FAl}^{-1}$

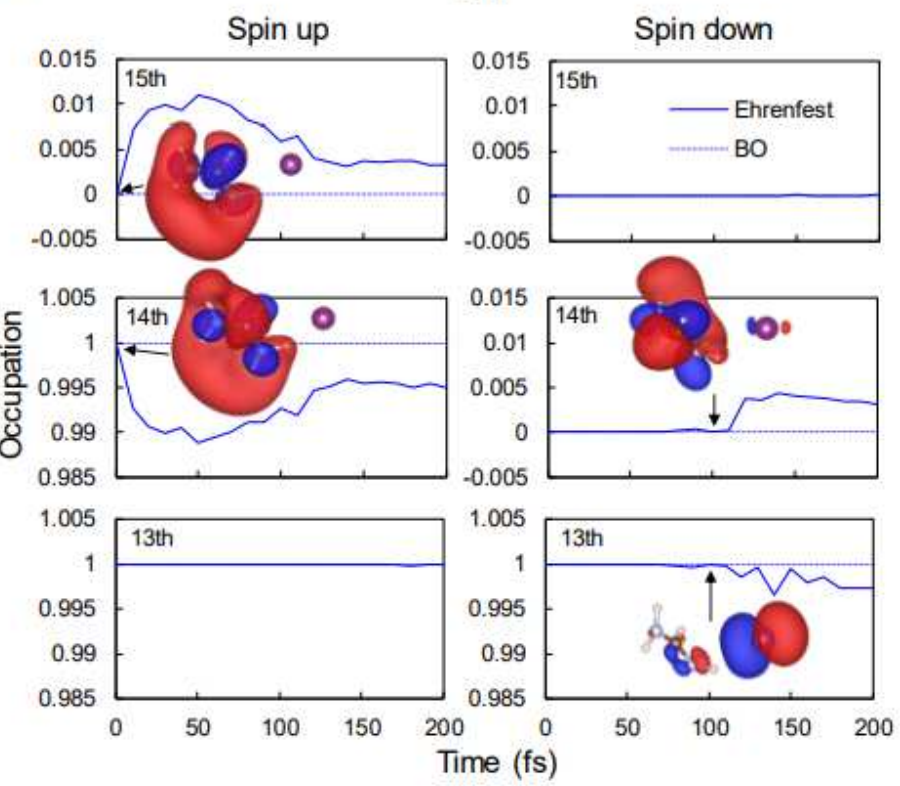

Figure 4

Time-dependent occupation of KS orbitals. a Occupation of KS orbitals of MAl-1. The 12th spin-up orbital corresponds to the anion HOMO of MAl-1 . Despite the non-adiabatic simulation, the occupation does not change as time passes. b Occupation of KS orbitals of FAl-1. The 14th spin-up orbital is the anion HOMO. Insets are the real parts of corresponding wavefunctions of the 14th and 15th spin-up states at $0 \mathrm{fs}$ and the 13th and 14th spin-down states at $100 \mathrm{fs}$.

a

$\mathrm{MAl}^{-1}$
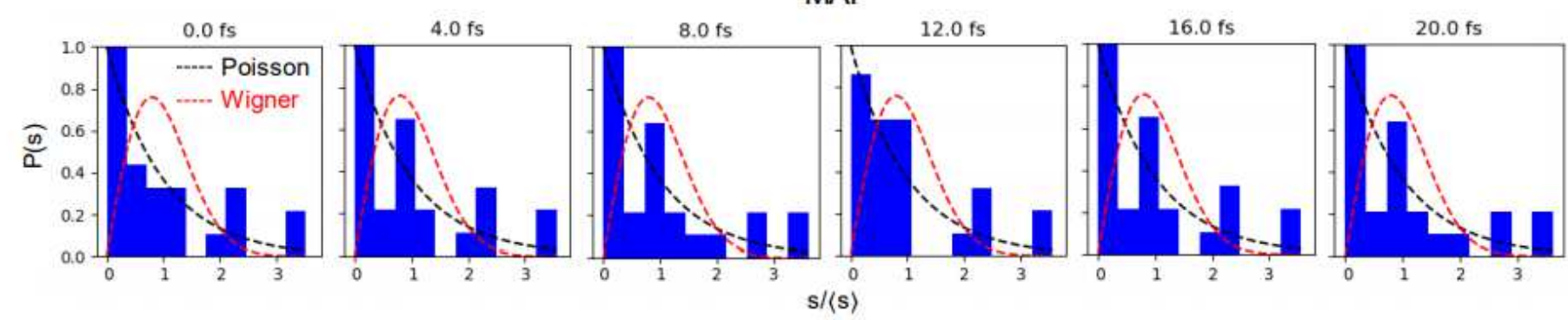

b

$\mathrm{FAl}^{-1}$
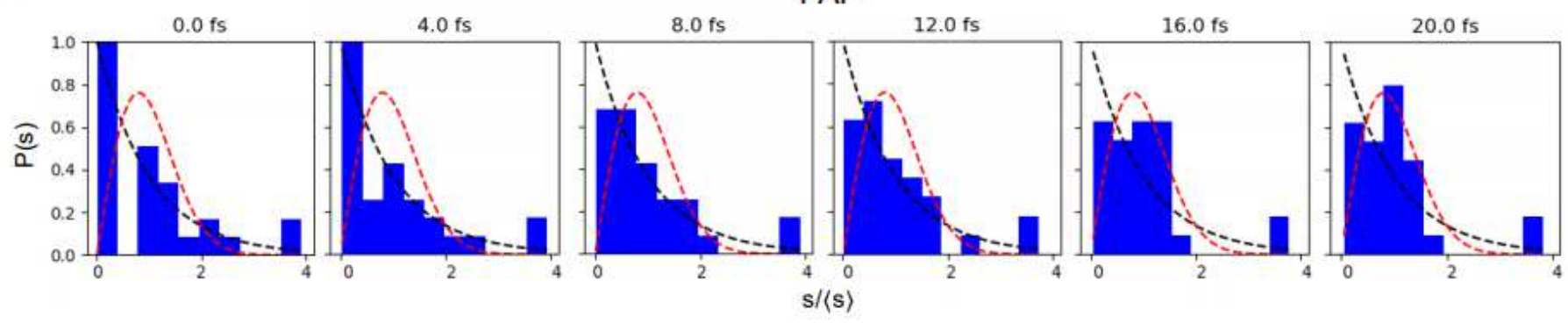


\section{Figure 5}

Level-spacing distribution of KS eigenvalues from 0 to $20 \mathrm{fs}$ in a MAl-1 and b FAl-1. In FAl-1, the distribution rapidly changes from Poisson to Wigner-Dyson statistics within $20 \mathrm{fs}$. A Poisson distribution

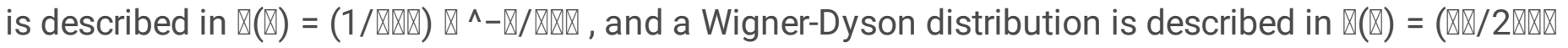

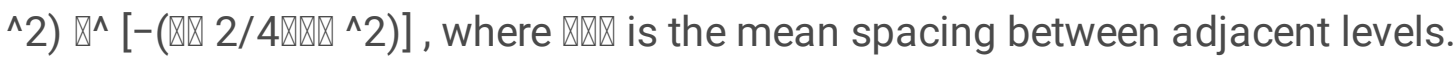

\section{Supplementary Files}

This is a list of supplementary files associated with this preprint. Click to download.

- SI.pdf 